\title{
ARTICLE
}

Received 24 Sep 2013 | Accepted 4 Mar 2014 | Published 10 Apr $2014 \quad$ DOl: 10.1038/ncomms4566

\section{Nucleic acid sensing by T cells initiates Th2 cell differentiation}

Takayuki Imanishi', Chitose Ishihara ${ }^{1}$, Mohamed El Sherif Gadelhaq Badr ${ }^{1}$, Akiko Hashimoto-Tane ${ }^{1}$, Yayoi Kimura ${ }^{2}$, Taro Kawai ${ }^{3, \dagger}$, Osamu Takeuchi ${ }^{3, \dagger}$, Ken J. Ishii ${ }^{4,5}$, Shun'ichiro Taniguchi ${ }^{6}$, Tetsuo Noda ${ }^{7}$, Hisashi Hirano ${ }^{2}$, Frank Brombacher ${ }^{8}$, Glen N. Barber ${ }^{9}$, Shizuo Akira ${ }^{3}$ \& Takashi Saito $^{1,10}$

While T-cell responses are directly modulated by Toll-like receptor (TLR) ligands, the mechanism and physiological function of nucleic acids (NAs)-mediated T cell costimulation remains unclear. Here we show that unlike in innate cells, T-cell costimulation is induced even by non-CpG DNA and by self-DNA, which is released from dead cells and complexes with antimicrobial peptides or histones. Such NA complexes are internalized by T cells and induce costimulatory responses independently of known NA sensors, including TLRs, RIG-I-like receptors (RLRs), inflammasomes and STING-dependent cytosolic DNA sensors. Such NA-mediated costimulation crucially induces Th2 differentiation by suppressing T-bet expression, followed by the induction of GATA-3 and Th2 cytokines. These findings unveil the function of NA sensing by T cells to trigger and amplify allergic inflammation.

\footnotetext{
${ }^{1}$ Laboratory for Cell Signaling, RCAI, RIKEN Center for Integrative Medical Sciences (IMS-RCAI), Yokohama, Kanagawa 230-0045, Japan. ${ }^{2}$ Graduate School of Medical Life Sciences, Yokohama City University, Yokohama, Kanagawa 236-0004, Japan. ${ }^{3}$ Laboratory of Host Defense, WPI Immunology Frontier Research Center, Osaka University, Suita, Osaka 565-0871, Japan. ${ }^{4}$ Laboratory of Adjuvant Innovation, National Institute of Biomedical Innovation, Ibaraki, Osaka 567-0085, Japan. ${ }^{5}$ Laboratories of Vaccine Science, WPI Immunology Frontier Research, Osaka University, Suita, Osaka 565-0871, Japan.

${ }^{6}$ Department of Molecular Oncology, Institute of Pathogenesis and Disease Prevention, Shinshu University Graduate School of Medicine, Matsumoto, Nagano 390-8621, Japan. ${ }^{7}$ Department of Cell Biology, Cancer Research Institute of the Japanese Foundation of Cancer Research, Toshima-ku, Tokyo 1708455, Japan. ${ }^{8}$ International Center for Genetic Engineering and Biotechnology, Cape Town Component and Institute of Infectious Diseases and Molecular Medicine, Faculty of Health Science, Division of Immunology, University of Cape Town, Cape Town, South Africa. ${ }^{9}$ Department of Cell Biology and the Sylvester Comprehensive Cancer Center, University of Miami Miller School of Medicine, Miami, Florida 33136, USA. ${ }^{10}$ Laboratory for Cell Signaling, WPI Immunology Frontier Research Center, Osaka University, Suita, Osaka 565-0871, Japan. † Present addresses: Laboratory of Molecular Immunobiology, Graduate School of Biological Sciences, Nara Institute of Science and Technology (NAIST), Ikoma, Nara 630-0192, Japan (T.K.); Laboratory of Infection and Prevention, Institute for Virus Research, Kyoto University, Sakyo-ku, Kyoto 606-8507, Japan (O.T.). Correspondence and requests for materials should be addressed to T.S. (email: saito@rcai.riken.jp).
} 
$\mathrm{T}$ oll-like receptors (TLRs) sense pathogen-associated molecular patterns to initiate not only innate responses but also to help regulate $\mathrm{T}$ cell-mediated adaptive immune responses ${ }^{1,2}$. While some TLRs are expressed on the cell surface, NA-sensing TLRs such as TLR3, TLR7/8 and TLR9 are expressed in endosomal compartments, allowing specific recognition of endocytosed pathogens.

Recent studies have shown that $\mathrm{T}$ cells also express TLRs and that TLR ligands can directly modulate T-cell responses. For example, TLR2 ligands directly promote proliferation of activated $\mathrm{T}$ cells $s^{3,4}$, modulate the proliferation and suppressive functions of $\mathrm{CD} 4{ }^{+} \mathrm{CD} 25^{+}$regulatory $\mathrm{T}$ cells $s^{5,6}$, trigger Th1 effector functions independently of TCR stimulation ${ }^{7}$ and modulate Th17 responses ${ }^{8}$.

In addition, it has been reported that ligands for NA-sensing TLRs enhance IL-2 production and proliferation of anti-CD3 antibody $(\mathrm{Ab})$-stimulated $\mathrm{T}$ cells $\mathrm{s}^{9,10}$ and promote survival of activated T cells ${ }^{11}$, and further that the TLR8 ligand inhibits the suppressive function of regulatory $\mathrm{T}$ cells ${ }^{12}$. However, except for TLR2, very little is known about the molecular basis of the NA-sensing mechanisms and the functional consequences of NA-mediated costimulation in T cells.

Naive $\mathrm{CD} 4{ }^{+} \mathrm{T}$ cells differentiate into various effector $\mathrm{T}$ helper (Th) cells such as Th1, Th2 and Th17 cells, which produce IFN- $\gamma$, IL-4/IL-5/IL-9/IL-13 and IL-17/IL-22, respectively ${ }^{13}$. While Th1 and Th17 cells exhibit protective functions against intracellular pathogens and extracellular bacteria/fungi, Th2 cells protect from helminthic infection. Contrary to these protective functions, the same Th subsets can play a role in disease pathogenesis: Th1 for inflammatory diseases, Th2 for allergic diseases and Th17 for autoimmune diseases. While TLR stimulation of antigen-presenting cells (APCs) results in the production of IL-12, which induces Th1 differentiation, Th2 development is induced by IL-4, but the cells responsible for the initial wave of IL-4 production needed to induce Th2 differentiation remain elusive ${ }^{14}$.

In this study, we report that NA-induced costimulatory responses of $\mathrm{CD}^{+}{ }^{+} \mathrm{T}$ cells are mediated independently of known NA sensors in innate immunity. We found that $\mathrm{T}$ cells take up NAs to induce costimulation and that the NA-mediated costimulation requires higher-order structure of the NAs by forming complexes with the antimicrobial peptides or with core histones. More importantly, costimulation of naive $\mathrm{CD} 4^{+} \mathrm{T}$ cell with NAs induces Th2 differentiation through the downregulation of T-bet and the upregulation of GATA-3 expression. Thus, NAs directly induce T-cell costimulation through a unique NA-sensing mechanism to trigger the initial IL-4 production for Th2 differentiation, which might be involved in triggering and amplification of allergic inflammation.

\section{Results}

TLR-independent NA-mediated costimulation of $\mathrm{CD}^{+}{ }^{+} \mathrm{T}$ cells. To elucidate the functional significance of NA stimulation, naive $\mathrm{CD}^{+} \mathrm{T}$ cells were stimulated with each TLR ligand. While none of the TLR ligands alone were able to induce cell proliferation or IL-2 production, proliferation and IL-2 production were selectively enhanced by Pam3 (TLR1/2), MALP-2 (TLR2/6), poly(I:C) (TLR3) and CpG-B (TLR9) with anti-CD3 stimulation (Fig. 1a).

We next determined whether this response is mediated by TLRs using mice deficient in both MyD88 and TRIF, which lack the capacity to respond to any of the known TLR ligands. Surprisingly, both poly(I:C) and CpG-B-mediated costimulation were normal in the MyD88/TRIF-doubly deficient $\mathrm{CD} 4{ }^{+} \mathrm{T}$ cells, whereas MALP-2 (TLR2)-mediated costimulation was completely abrogated (Fig. 1b), demonstrating that poly(I:C) and CpG-B-mediated T-cell costimulation was induced independently of TLR signaling.

Notably, DNA lacking CpG motifs required for TLR9 activation, such as non-CpG oligodeoxynucleotide (ODN) and DNA encoding GFP (GFP-S and GFP-AS, antisense strand) could also induce costimulation for IL-2 production (Fig. 1c). These data indicate that DNA induces T-cell costimulation independently of the CpG motifs. We also found that poly $(\mathrm{dA})$, poly $(\mathrm{dC})$ and poly $(\mathrm{dG})$ but not poly(dT) induced the costimulation for IL-2 production, although the uptake of poly $(\mathrm{dT})$ and non-CpG ODN by $\mathrm{T}$ cells was comparable (Fig. 1d).

Confocal microscopy analysis revealed that non-CpG ODN colocalized with an endosomal marker dextran and a lysosomal marker LysoTracker (Fig. 1e), indicating that non-CpG ODN is taken up by $\mathrm{T}$ cells and transported to endosomes/lysosomes, similarly in innate cells, and induces costimulatory signals in a TLR-independent manner.

Higher-order structure of NA induces T-cell costimulation. It is noteworthy that CpG-A possessing a poly(dG)-tail induced stronger costimulation of $\mathrm{CD} 4{ }^{+} \mathrm{T}$ cells than other ODNs such as CpG-B and non-CpG (Fig. 1c). The importance of the poly(dG) tract was confirmed by the finding that control ODN GpC corresponding to $\mathrm{CpG}-\mathrm{A}$ induces robust IL-2 production, similar to CpG-A (Fig. 2a), and that replacement of the poly(dG) motif of $\mathrm{GpC}$ by poly $(\mathrm{dA})$, poly $(\mathrm{dC})$ or poly $(\mathrm{dT})$ resulted in the complete loss of stimulatory activity (Fig. 2b).

It has been reported that the poly $(\mathrm{dG})$ tail induces the spontaneous formation of large multimeric aggregates via G-quadruplex formation ${ }^{15}$. Indeed, when $\mathrm{GpC}$ was rendered single-stranded by heating and flash-cooling, a dramatic reduction of IL-2 production was observed (Fig. 2c), suggesting that higher-order structures mediated by the poly $(\mathrm{dG})$ motif are critical for enhanced costimulation by CpG-A and GpC. Consistently, introduction of a poly(dG)-tail to non-CpG and CpG-B that possess a phosphodiester (PO) backbone sensitive to DNase enabled them to induce costimulation, whereas the same DNA without the poly(dG)-tail could not (Fig. 2d).

It has been shown that the poly $(\mathrm{dG})$ motif not only protects against DNase degradation ${ }^{16}$ but also enhances the cellular uptake of the ODN ${ }^{17}$. Indeed, the uptake of $\mathrm{GpC}-\operatorname{poly}(\mathrm{dC})$ by $\mathrm{T}$ cells was lower than $\mathrm{GpC}$, indicating that $\mathrm{GpC}$-poly $(\mathrm{dC})$ could not induce costimulation due to its poor uptake (Fig. 2e). It is noteworthy that cellular uptake of $\mathrm{GpC}$-poly $(\mathrm{dC})$ was weaker than that of non-CpG (Fig. 2e). It has been reported that phosphorothioate (PS)-modified ODN are taken up more efficiently than PO-ODN ${ }^{17}$. The entire backbone of non-CpG is PS, whereas it is only partial in GpC (Supplementary Table 1). PS modification of $\mathrm{GpC}$-poly $(\mathrm{dC})$ led to enhanced cellular uptake and costimulation (Fig. 2e). By contrast, modification of nonCpG to contain only partial PS resulted in decreased uptake and the failure of costimulation (Fig. 2e). We also confirmed that cellular uptake and costimulation of PO-backboned non-CpG was much weaker than those of PS-backboned non-CpG (Supplementary Fig. 1a). These data indicate that efficient uptake of DNA via its poly(dG) tail or PS modification is critical for induction of costimulation.

While inosine or guanine-containing RNA such as poly(I), poly(G), poly(I:C) and poly(C:G) could induce costimulation, poly(A), poly(U), poly(C) and poly(A:U) could not (Fig. 2f). The induction of costimulation by RNA was correlated with the cellular uptake of RNA (Fig. 2f). These data indicate that RNA-mediated costimulation depends on the RNA sequence for cellular uptake of RNA to induce costimulation. 
a

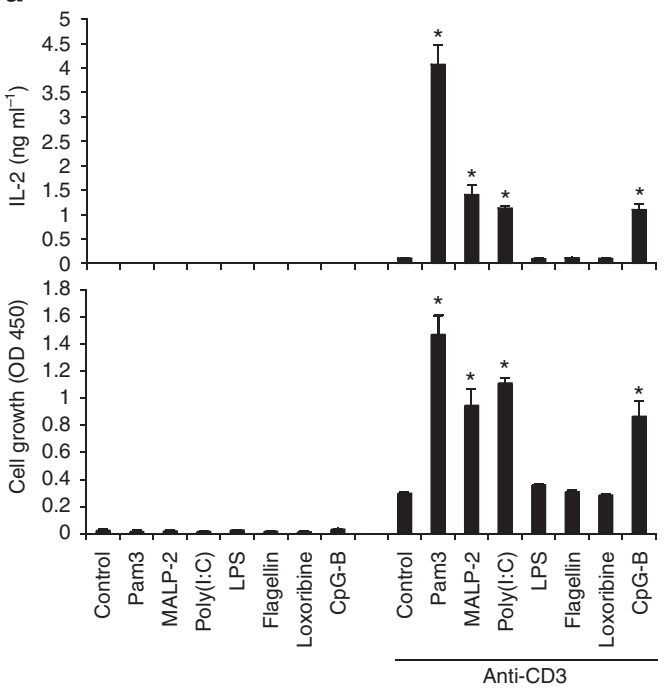

C

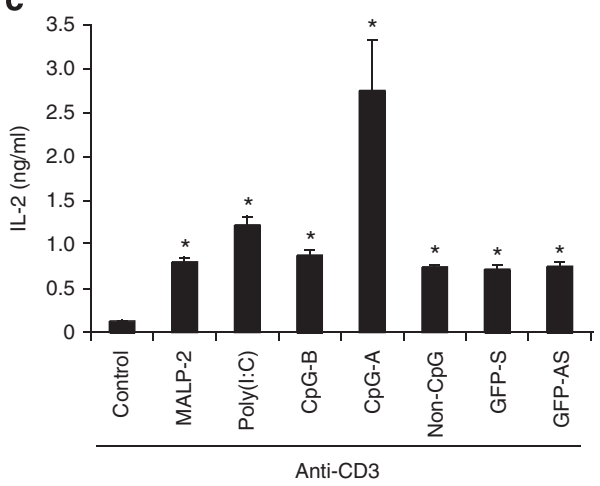

b

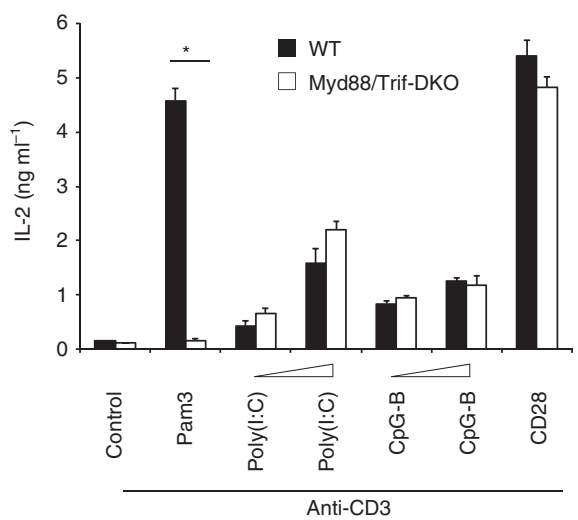

d

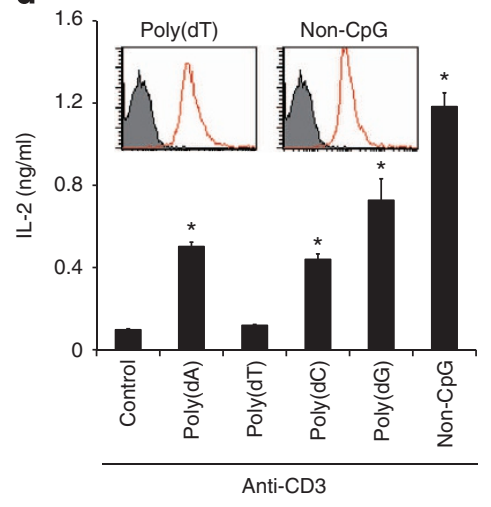

e

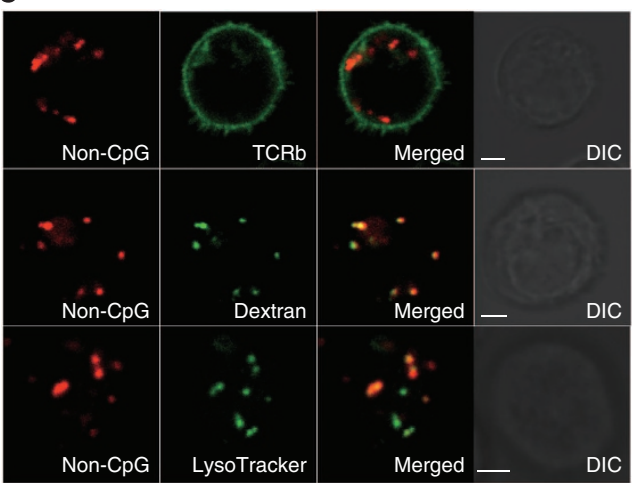

Figure 1 | DNA/RNAs are incorporated into and induce costimulation of CD4 ${ }^{+} \mathbf{T}$ cells. (a) Naive CD4 ${ }^{+}$T cells were cultured with indicated TLR ligands (Pam3: $5 \mu \mathrm{g} \mathrm{ml}^{-1}$, MALP-2: $5 \mu \mathrm{g} \mathrm{ml}{ }^{-1}$, poly(I:C): $100 \mu \mathrm{g} \mathrm{ml}^{-1}$, Flagellin: $1 \mu \mathrm{g} \mathrm{ml}{ }^{-1}$, Loxoribine: $100 \mu \mathrm{M}, \mathrm{CpG}-\mathrm{B}: 5 \mu \mathrm{M}$ ) in the presence or absence of immobilized anti-CD3€ Ab $\left(10 \mu \mathrm{g} \mathrm{ml}^{-1}\right)$. After 48-hour incubation, IL-2 production and cell growth were assessed by ELISA and MTS assay, respectively. ${ }^{\star} P<0.05$, Student's $t$-test (compared with anti-CD3 alone). (b) Naive CD4 ${ }^{+}$T cells from WT or Myd88 ${ }^{-/-}$Trif ${ }^{-/-}$mice were stimulated with the indicated TLR ligands or anti-CD28 (Clone: $37.51,5 \mu \mathrm{g} \mathrm{ml}^{-1}$ ) in the presence of immobilized anti-CD3€ Ab. ${ }^{\star} P<0.05$, Student's $t$-test (compared with WT cells treated with Pam3). (c,d) Naive CD4 ${ }^{+}$T cells were stimulated with the indicated NAs (c) and ODNs (d) in the presence of immobilized anti-CD3€ Ab. These T cells were incubated with the Cy5-labelled ODNs at $37^{\circ} \mathrm{C}$ for 90 min and ODN uptake was determined by flow cytometry (d, upper). ${ }^{\star} P<0.05$, Student's $t$-test (compared with anti-CD3 alone). (e) Naive CD4 ${ }^{+} \mathrm{T}$ cells were incubated with $5 \mu \mathrm{M}$ non-CpG-Cy5 for $90 \mathrm{~min}$ and dextran-Alexa Fluor 488 or LysoTracker for last $10 \mathrm{~min}$ for the subcellular staining of endosomes and lysosomes, respectively. Confocal microscopy data with differential interference contrast (DIC) images of representative cells are shown. Scale bars, $2.5 \mu \mathrm{m}$. Error bars indicate s.d. Data are representative of at least three independent experiments.

It has been reported that poly(I) forms parallel four-stranded helices held together by hydrogen-bonded inosine quartets, similar to poly $(\mathrm{dG})$ chains $^{18}$. We found that heat denaturation of poly(I:C) and poly $(\mathrm{G})$ resulted in impaired costimulation (Fig. 2g), indicating that the ability of RNA to induce T-cell costimulation is dependent on the higher-order structure similar to DNA. 

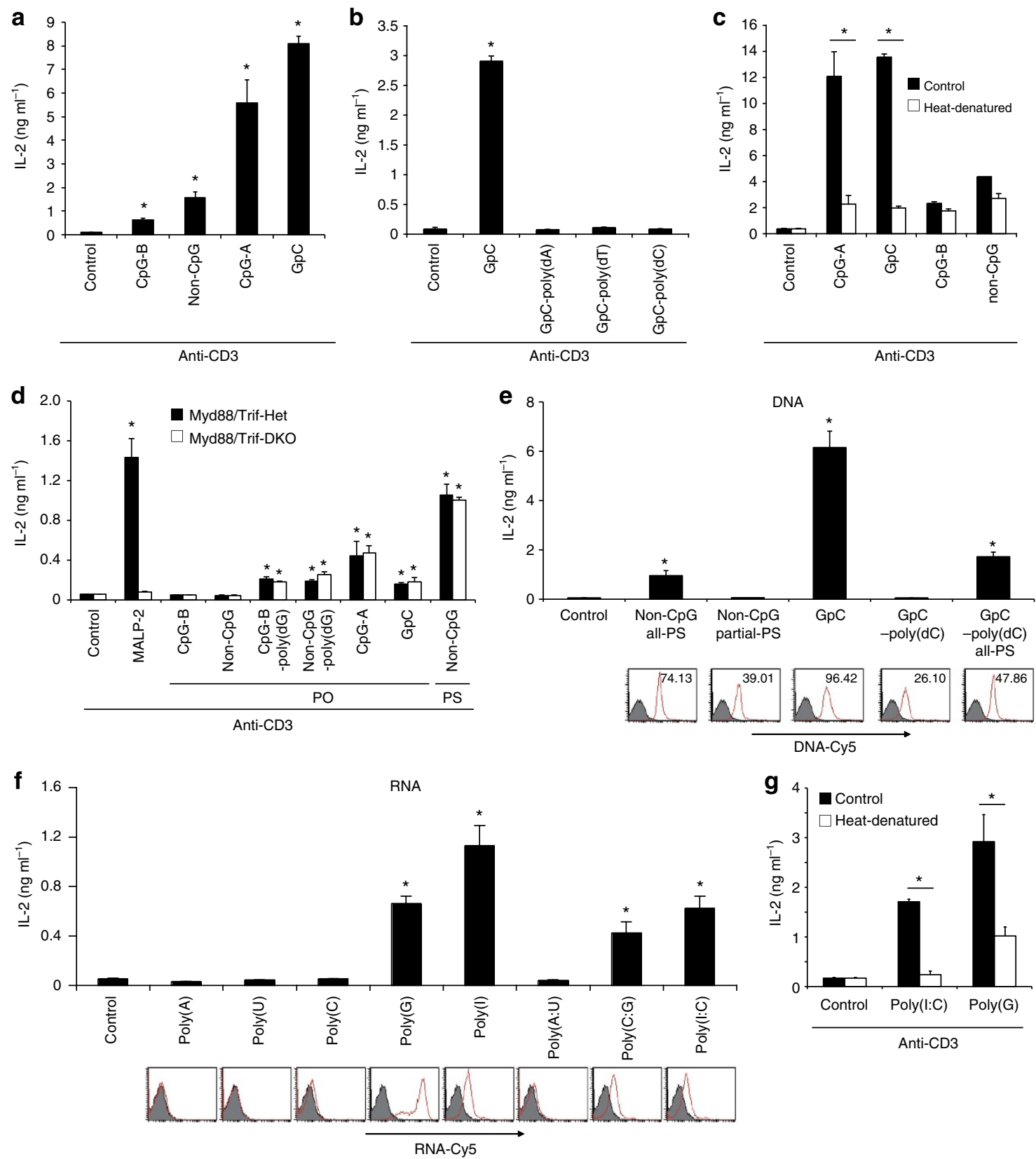

Figure 2 | Properties of DNA/RNAs required for T cell costimulatory activity. (a-c) Naive CD4 ${ }^{+} \mathrm{T}$ cells were activated with plate-bound anti-CD3e with various DNAs $-{ }^{\star} P<0.05$, Student's $t$-test (compared with anti-CD3 alone) (a); GpC with different poly tails. ${ }^{*} P<0.05$, Student's $t$-test (compared with anti-CD3 alone) (b); and heat-denatured ODNs. ${ }^{\star} P<0.01$, Student's $t$-test (compared with the untreated control) (c). After $48 \mathrm{~h}$, IL-2 production was measured by ELISA. (d) Naive CD4 ${ }^{+}$T cells derived from Myd88 $8^{+/-}$Trif $^{+/-}$and Myd88 $8^{-/-}$Trif $^{-/-}$mice were activated with immobilized anti-CD3€ together with the indicated DNAs. ${ }^{\star} P<0.05$, Student's $t$-test (compared with anti-CD3 alone in WT cells). (e) Naive CD4 ${ }^{+}$T cells were stimulated with immobilized anti-CD3€ together with all (all-PS) or partially (partial-PS) PS-modified ODN. CD4 ${ }^{+} \mathrm{T}_{\text {cells }}$ were incubated with the Cy5-labelled ODN at $37^{\circ} \mathrm{C}$ for $90 \mathrm{~min}$, washed and analysed for incorporated ODN by flow cytometry (bottom panels). ${ }^{\star} P<0.05$, Student's $t$-test (compared with anti-CD3 alone). (f) Naive CD4 ${ }^{+}$T cells were stimulated with indicated ODNs and analysed similarly as in (e). ${ }^{\star} P<0.05$, Student's $t$-test (compared with anti-CD3 alone). (g) Naive CD4 ${ }^{+}$T cells were stimulated and analysed similarly in (c). ${ }^{\star} P<0.05$, Student's $t$-test (compared with the untreated control). Error bars indicate s.d. Data are representative of at least three independent experiments.

Costimulation induced by NA complexed with LL37 and histones. It has been recently shown that endogenous self-DNAs stimulate plasmacytoid dendritic cells by forming aggregated structures upon binding with the antimicrobial peptide LL37 (ref. 19). Similarly, we found that mammalian and bacterial genomic DNA were taken up by $\mathrm{T}$ cells and induced costimulation when mixed with LL37 while they alone were neither incorporated nor induce stimulation (Supplementary
Fig. 2a, Fig. 3a). Similarly, although poly(A) and poly(A:U) per se were defective in cellular uptake and induction of costimulation of naive $\mathrm{CD} 4{ }^{+} \mathrm{T}$ cells (Fig. 2f), they were incorporated and induced T-cell costimulation when complexed with LL37 (Supplementary Fig. 2b, Fig. 3b).

Similar to LL37, extracellular histones as components of neutrophil extracellular traps exhibit antimicrobial function ${ }^{20}$. We found that the addition of core histones $(\mathrm{H} 2 \mathrm{~A}, \mathrm{H} 2 \mathrm{~B}, \mathrm{H} 3$ and 
a
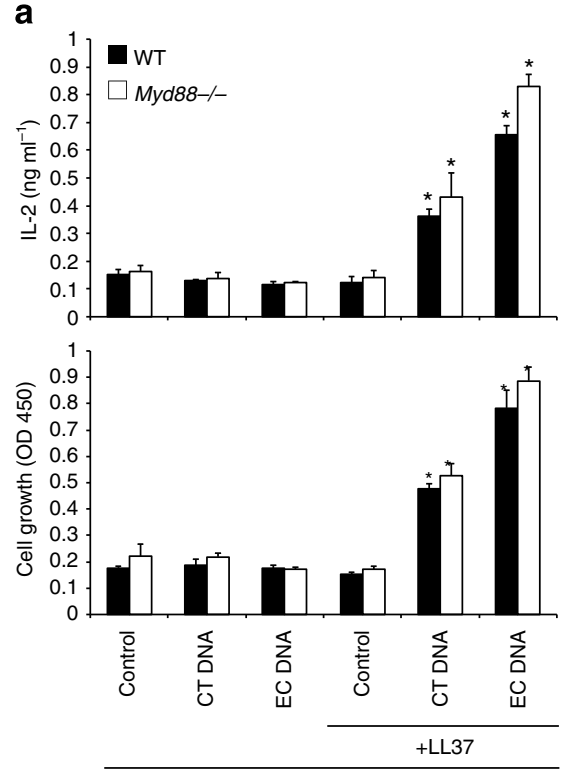

Anti-CD3 b

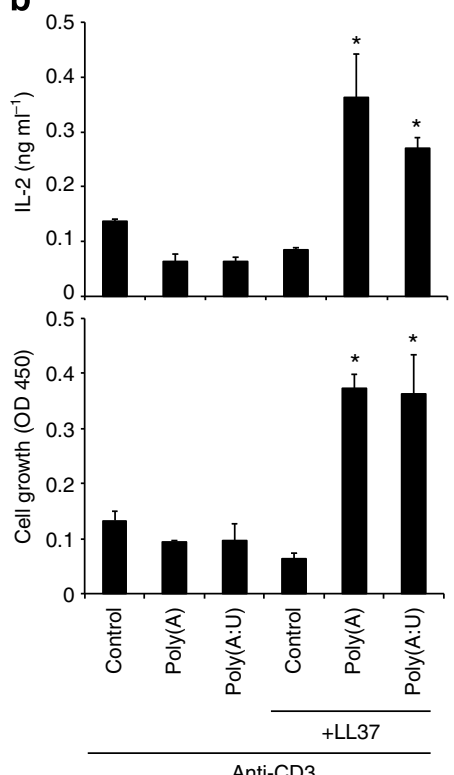

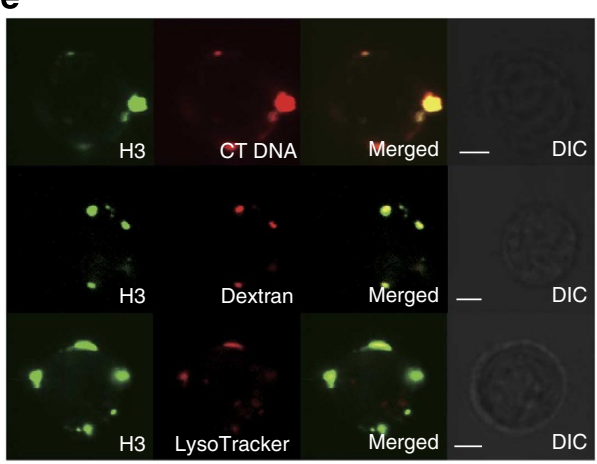

f

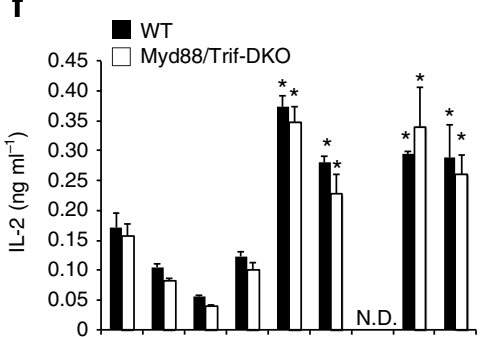

Control
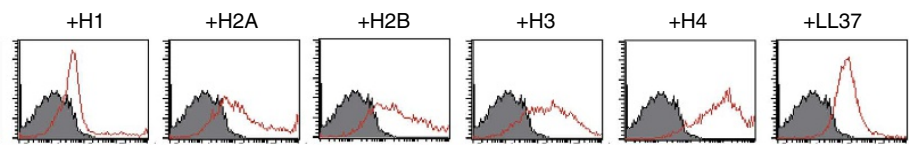

CT DNA

d

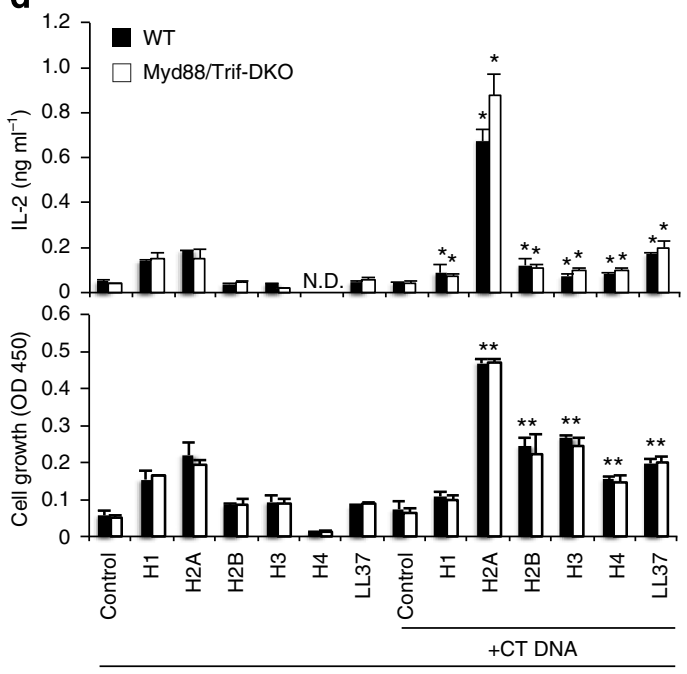

Anti-CD3

Figure 3 | T-cell activation by NAs complexed with antimicrobial peptides or core histones. (a,b) Naive CD4 ${ }^{+}$T cells derived from WT and Myd88-/mice were stimulated with plate-bound anti-CD3€ together with calf thymus (CT)- or E. coli (EC)-derived genomic DNA (a) or RNAs (b) either alone or premixed with LL37. After $48 \mathrm{~h}, \mathrm{IL}-2$ production and cell growth were assessed by ELISA and MTS assay, respectively. ${ }^{\star} P<0.05$, Student's $t$-test (compared with anti-CD3 plus LL37 in WT cells). (c) Naive CD4 ${ }^{+}$T cells were incubated with the Cy5-labelled CT DNA premixed with the indicated histones or LL37 at $37^{\circ} \mathrm{C}$ for 90 min and the incorporated DNA were analysed by flow cytometry. (d,f) Naive CD4 ${ }^{+}$T cells derived from WT and Myd88-/- Trif ${ }^{-/-}$mice were stimulated with plate-bound anti-CD3 together with CT DNA alone (d), RNAs alone (f) or premixed with various histones (d,f), and analysed similarly in (a). ${ }^{*} P<0.05$, Student's $t$-test (compared with anti-CD3 plus each histone or LL37 in WT cells). (e) Naive $\mathrm{CD}^{+}{ }^{+} \mathrm{T}$ cells were stimulated with anti-CD3 with Cy5-labelled CT DNA premixed with Alexa488-labelled histone H3 for $18 \mathrm{~h}$. Endosomes and Lysosomes were visualized by staining with dextran-Alexa Fluor 488 and LysoTracker, respectively. Confocal and differential interference contrast (DIC) images of representative cells are shown. Scale bars, $2.5 \mu \mathrm{m}$. Error bars indicate s.d. Data are representative of at least two independent experiments.

$\mathrm{H} 4$ ), but not the linker histone $\mathrm{H} 1$, increase cellular uptake of genomic DNA into $\mathrm{CD} 4^{+} \mathrm{T}$ cells (Fig. 3c). The uptake was correlated with induction of costimulation (Fig. 3d). Although the genomic DNA-H2A complex was the strongest inducer for IL-2 production, $\mathrm{H} 2 \mathrm{~A}$ itself induces costimulation in the absence of DNA through unknown mechanism. Therefore, we use H3 that has no costimulatory activity by itself to determine the localization of histone/DNA complexes. The genomic 
DNA-histone $\mathrm{H} 3$ complex was incorporated and localized in endosomes and lysosomes as shown by colocalization with dextran and LysoTracker, respectively (Fig. 3e). Similarly to LL37, histone $\mathrm{H} 3$ and $\mathrm{H} 4$ allowed $\operatorname{poly}(\mathrm{C})$ and $\operatorname{poly}(\mathrm{A}: \mathrm{U})$ to induce costimulation of naive $\mathrm{CD}^{+} \mathrm{T}$ cells (Fig. 3f). These data indicated that NAs from self or pathogens could induce T-cell costimulation by forming complexes with antimicrobial peptides such as LL37 or core histones.

NA-mediated costimulation is independent of known sensors. To determine the mechanism of NA recognition and activation in $\mathrm{T}$ cells, we analysed the possible involvement of cytosolic sensors of NAs in innate cells. DNA-dependent activator of IRFs (DAI; also known as ZBP1) was first reported to function as a cytoplasmic DNA receptor ${ }^{21}$. Absent in melanoma 2 (AIM2) was identified as a cytosolic DNA sensor that activates inflammasome ${ }^{22}$. Stimulator of IFN genes (STING) and TBK1 have been identified as essential molecules that mediate a wide range of cytosolic DNA-induced type I IFN responses ${ }^{23-25}$. To examine the possible involvement of these sensors for $\mathrm{T}$-cell costimulation, we tested naive $\mathrm{CD}^{+}{ }^{+} \mathrm{T}$ cells derived from $Z_{b p 1^{-/-}}, A s c^{-/-}$(which links AIM2 to caspase-1), Sting ${ }^{-/-}$ and $T n f^{-/-} T b k 1^{-/}$mice. However, surprisingly, DNAmediated costimulation was induced normally in these $\mathrm{T}$ cells
(Fig. 4a-c, Supplementary Fig. 3a), strongly suggesting that T cells utilize a DNA-sensing system different from innate immune cells.

TLR3 recognizes poly(I:C) in the endosome and initiates signalling through the adaptor, $\mathrm{TRIF}^{1}$. On the other hand, retinoic acid-inducible gene I (RIG-I) and melanoma differentiation-associated gene 5 (MDA5) sense poly(I:C) and viral RNA in the cytoplasm, which activates an adaptor, IFN- $\beta$ promoter stimulator 1 (IPS-1; also known as MAVS) ${ }^{26,27}$. To examine the possibility that RIG-I/MDA5 and TLR3 may recognize RNA cooperatively or separately in $T$ cells, we examined RNA-mediated $\mathrm{T}$ cell costimulation in IPS-1/TRIFdoubly deficient mice. Normal costimulation by poly(I:C) and poly(I) was observed in Ips-1 ${ }^{-/}-$Trif $^{-/}-\mathrm{T}$ cells (Fig. 4d). In addition, to determine the functional redundancy between TLRs and inflammasomes or RIG-I-like receptors (RLRs), we generated MyD88/ASC-and MyD88/IPS-1 doubly-deficient mice. NA-mediated costimulation was normally induced in naive CD4 ${ }^{+}$ $\mathrm{T}$ cells from both mutant mice (Supplementary Fig. 3b,c). It has been demonstrated that NAs are promiscuously sensed by HMGB proteins to induce type I IFN and pro-inflammatory cytokines $^{28}$. However, downmodulation of all three HMGB proteins in $\mathrm{CD}^{+} \mathrm{T}$ cells using small interfering RNA did not alter IL-2 production in response to NAs (Supplementary Fig. 3d).

To identify the mechanism underlying the NA-mediated costimulatory signal to induce IL-2 production in $\mathrm{T}$ cells, we
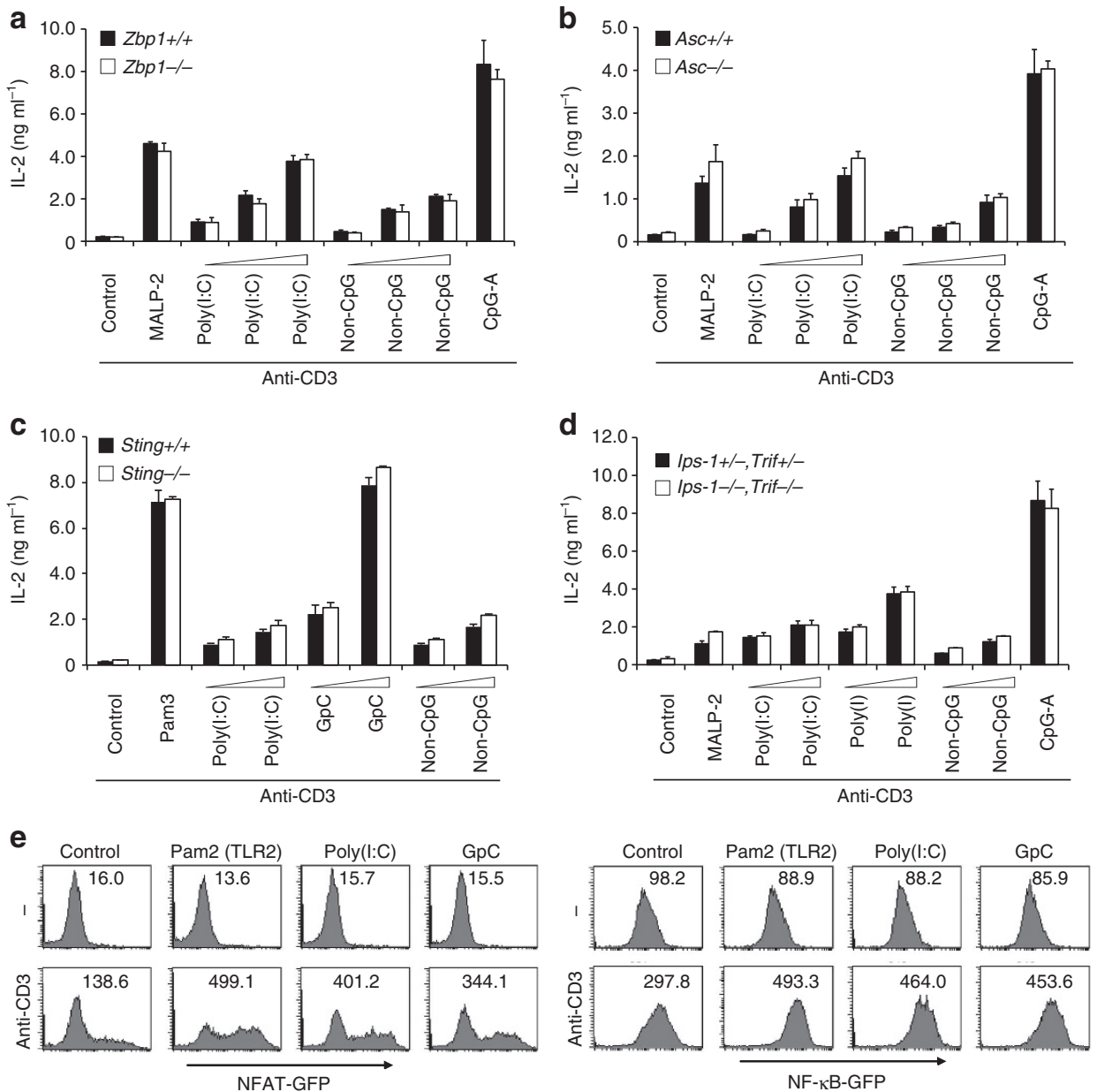

Figure 4 | NA-mediated T cell costimulation is independent of known innate sensors. (a-d) Naive $\mathrm{CD} 4^{+} \mathrm{T}_{\text {cells }}$ derived from WT or $Z b p 1^{-/-}$ (a), Asc $^{-/-}$(b), Sting ${ }^{-/-}$(c) or Ips-1 ${ }^{-/-}$Trif $^{-/-}$(d) mice were stimulated with plate-bound anti-CD3€ and the indicated ligands. After $48 \mathrm{~h}$, IL-2 production was measured by ELISA. (e) T-cell hybridoma reporter cells expressing NFAT-GFP (left) or NF-кB-GFP (right) were stimulated with the indicated ligands with or without immobilized anti-CD3€ for $24 \mathrm{~h}$ and analysed by flow cytometry. Error bars indicate s.d. Data are representative of at least two independent experiments. 
a

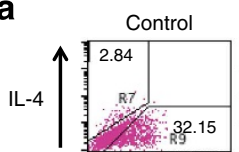
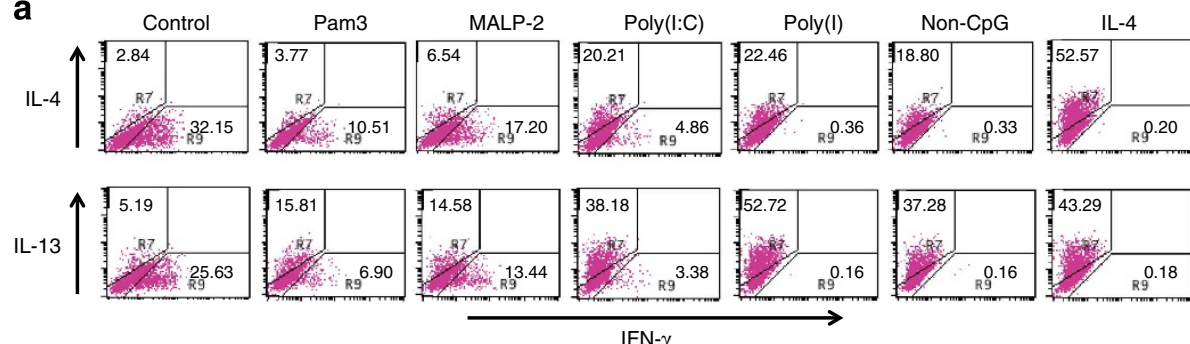

b
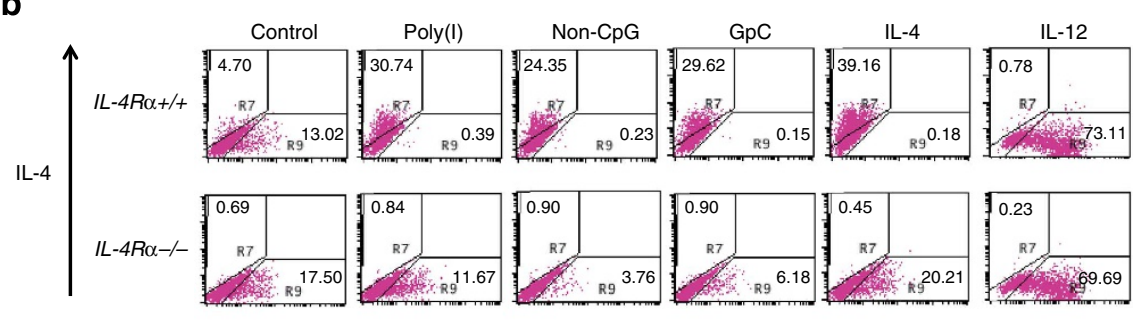
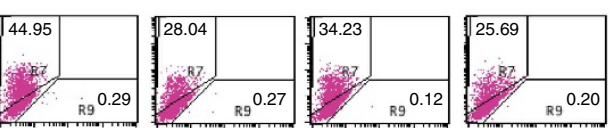

IL-13
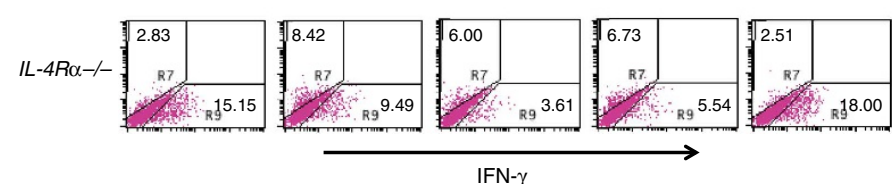

IFN- $\gamma$

$\mathbf{e}$
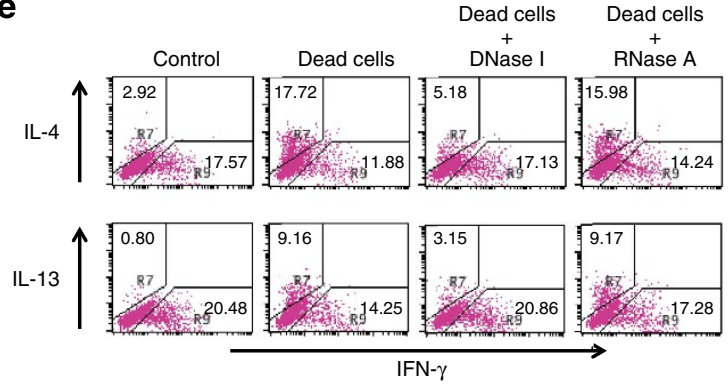

f

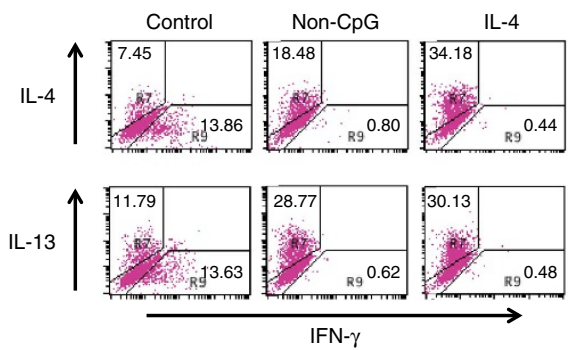

C

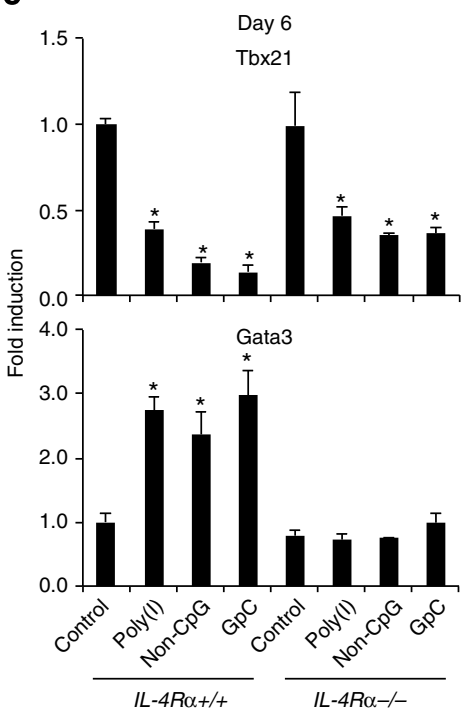

d
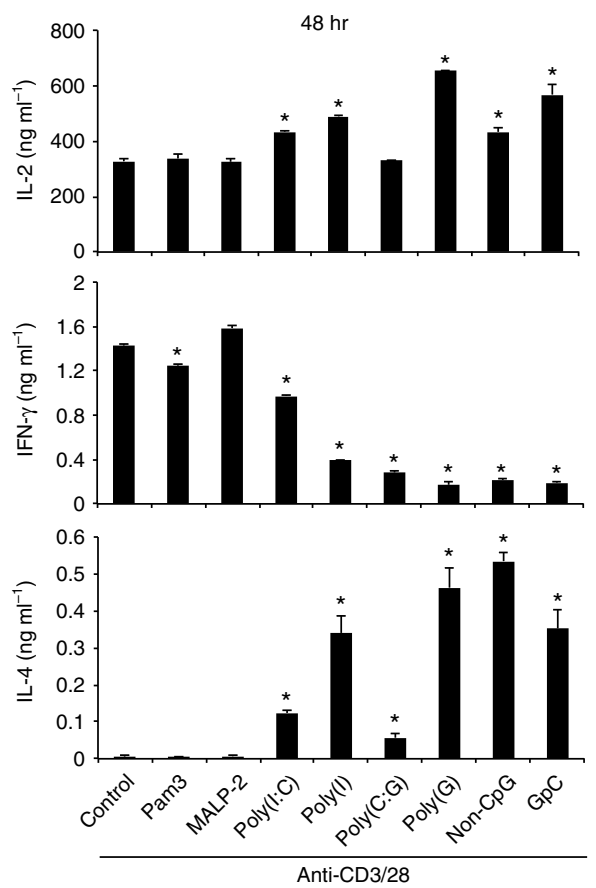

Figure 5 | NA-mediated costimulation induces Th2 differentiation. (a) Naive $\mathrm{CD}^{+}{ }^{+} \mathrm{T}$ cells were stimulated with immobilized anti-CD3€ $\left(10 \mu \mathrm{g} \mathrm{ml}^{-1}\right)$ plus anti-CD28 $\left(10 \mu \mathrm{g} \mathrm{ml}^{-1}\right)$ and the indicated ligands for 3 days, followed by culture with IL-2 $\left(10 \mathrm{ng} \mathrm{ml}^{-1}\right)$ for an additional 3 days. Cells were subjected to real-time PCR analysis (b), or restimulated with immobilized anti-CD3€ plus anti-CD28 for $6 \mathrm{~h}$ for staining of intracellular cytokines IL-4 and IL-13. (b,c) Naive CD4 ${ }^{+}$T cells from WT and IL-4R $\alpha^{-/-}$mice were activated similarly as in (a) and cells were subjected to real-time PCR analysis (c) or restimulated with immobilized anti-CD3€ plus anti-CD28 for $6 \mathrm{~h}$ for staining of intracellular cytokines IL-4 and IL-13. ${ }^{\star} P<0.05$, Student's $t$-test (compared with anti-CD3/CD28 plus non-CpG in WT cells). (d) Naive CD4 ${ }^{+}$T cells were stimulated for $48 \mathrm{~h}$ with anti-CD3€ plus anti-CD28 and the indicated ligands and cytokine production was analysed by ELISA. ${ }^{\star} P<0.05$, Student's $t$-test (compared with anti-CD3/CD28 alone). (e) Naive $\mathrm{CD} 4{ }^{+}$T cells with or without $\gamma$-irradiated naive CD4 ${ }^{+}$T cells (Dead cells) at a 1:1 ratio were stimulated with anti-CD3€ plus anti-CD28 in the presence or absence of DNase I or RNase A, and analysed similarly as in (a). (f) Naive CD4 ${ }^{+}$T cells from OT-II Tg mice were co-cultured with irradiated T cell-depleted splenocytes from C57BL/ 6 mice as APCs, together with OVA peptide $(10 \mu \mathrm{M})$ in the presence or absence of non-CpG for 6 days. CD4 ${ }^{+} \mathrm{T}$ cells were restimulated with immobilized anti-CD3€ plus anti-CD28 for $6 \mathrm{~h}$ for staining of intracellular cytokines. Error bars indicate s.d. Data are representative of at least three independent experiments.

analysed the activation of NF- $\mathrm{KB}$ and NFAT, both of which are essential for T-cell activation ${ }^{29}$. T-cell hybridoma (2B4) expressing the NF-KB-GFP or NFAT-GFP was stimulated by NAs plus anti-CD3. We observed that poly(I:C) and GpC markedly increased activation of NF- $\mathrm{BB}$ and NFAT, compared with anti-CD3 alone (Fig. 4e). These data suggest that enhanced 
activation of NF- $\kappa \mathrm{B}$ and NFAT is involved in the induction of NA-mediated T-cell costimulation. It is also worth noting that NA-induced costimulation of a T-cell hybridoma, which is definitely free of any innate cells, confirms that NA directly stimulates $\mathrm{T}$ cells.

NA-mediated costimulation induces Th2 cell differentiation. A recent study demonstrated that DNA released from dying host cells stimulates Th2 responses in vivo ${ }^{30}$. It is also reported that stimulation of RLRs by specific ligands biases the immune system toward a Th2 response, whereas the TLR signalling strongly induces Th1 and Th17 responses ${ }^{31}$. Accordingly, it seemed possible that NA-induced stimulation of T cells would induce Th2 cell differentiation. To test this hypothesis, naive $\mathrm{CD} 4{ }^{+} \mathrm{T}$ cells were stimulated in vitro with anti-CD3 plus anti-CD28 together with various NAs without blocking Abs against IFN- $\gamma$ or IL-4. NAs such as poly(I:C), poly(I) and non-CpG strongly induced the differentiation of IL-4-producing $\mathrm{T}$ cells without the addition of exogenous IL-4 (Fig. 5a). By contrast, NAs strongly inhibited the differentiation of IFN- $\gamma$-producing T cells (Fig. 5a), and also increased the frequency of Th2 cells producing IL-5, IL-9, IL-13 and IL-10 (Fig. 5a, Supplementary Fig. 4a). Consistently, the expression of the Th2-master regulator GATA-3 was enhanced, whereas the expression of T-bet, the Th1-master regulator was strongly inhibited in the T cells cultured with NAs (Supplementary Fig. 4b), suggesting that NAs directly induce the differentiation of Th2 cells. Notably, unlike NAs, TLR2 ligands had a minimal effect on Th1 and Th2 differentiation (Fig. 5a), and NA-mediated costimulation did not affect the development of IL-17-producing T cells (Supplementary Fig. 4c). Since NAs induced Th2 differentiation of MyD88/TRIF-double deficient $\mathrm{T}$ cells similarly to control $\mathrm{T}$ cells (Supplementary Fig. 4d), this processes does not require TLR signalling.

The generation of Th2 cells is dependent on IL-4-STAT6 signalling, which leads to the upregulation of GATA-3 (ref. 32). We assessed whether Th2 differentiation induced by NA-mediated costimulation was also IL-4-STAT6-dependent. IL-4 receptor (R) $\alpha$-deficient T cells after activation by NAs for 6 days failed to produce any Th2 cytokines including IL-4 and IL-13 (Fig. 5b). In addition, the induction of GATA-3 expression was severely diminished in IL- $4 \mathrm{R} \alpha$-deficient $\mathrm{T}$ cells (Fig. $5 \mathrm{c}$ ). Similar results were obtained using Stat $^{-/-} \mathrm{CD} 4{ }^{+} \mathrm{T}$ cells (Supplementary Fig. 4e), indicating that NA-mediated Th2 differentiation requires IL-4-STAT6 signalling. However, NAmediated inhibition of IFN- $\gamma$ production and T-bet expression was still observed in IL- $4 \mathrm{R} \alpha$-deficient $\mathrm{T}$ cells (Fig. 5b,c), suggesting that NA-mediated inhibition of Th1 differentiation is independent of IL-4 signalling. Therefore, it is likely that NA-mediated costimulation may enhance IL-4 production at an early time point (within $48 \mathrm{~h}$ ), which then induces Th2 differentiation. Indeed, IL- 4 production was induced at $48 \mathrm{~h}$ by NAs but not TLR2 ligands, whereas IFN- $\gamma$ production was reduced (Fig. 5d). NA-induced enhancement of IL-2 production was not strong upon stimulation with anti-CD3 plus CD28 as compared with anti-CD3 alone. Strong costimulation with anti-CD28 resulted in reduced enhancement, though significantly enhanced (Fig. 5d).

Collectively, these data demonstrate that NAs induce Th2 differentiation in an IL-4 signal-dependent manner similarly to the canonical Th2 differentiation pathway induced by exogenous IL-4, whereas suppression of Th1 differentiation by NAs is independent of IL-4 signalling.

We next determined whether self-DNA from dead cells induce Th2 differentiation. We found that the addition of dead cells (irradiated naive CD4 ${ }^{+}$T cells or irradiated HEK 293 cells) to the T-cell culture enhanced the differentiation of IL-4-producing cells, which was cancelled by the addition of DNase I into the medium (Fig. 5e, Supplementary Fig. 5f). Addition of RNase A resulted in minimal effect. These data suggest that self-DNA is a critical factor for Th2 differentiation induced by dead cells. To examine whether NA-mediated Th2 differentiation is induced upon antigen stimulation, OVA-specific naive CD4 $+\mathrm{T}$ cells from OT-II Tg mice were stimulated with OVA peptide-pulsed irradiated splenocytes plus non-CpG. Non-CpG promoted Th2 differentiation under the condition, strongly suggesting that NAs induces $\mathrm{T}$-cell costimulation even when $\mathrm{T}$ cells are activated by antigen-pulsed APCs (Fig. 5f).

Mechanisms underlying NA-induced Th2 differentiation. To determine the mechanism by which NAs induces Th2 differentiation, we compared the gene expression profiles in $\mathrm{CD} 4{ }^{+} \mathrm{T}$ cells activated under neutral conditions in the presence or absence of non-CpG. Surprisingly, before the upregulation of GATA-3, the expression of T-bet was strongly inhibited by nonCpG-mediated costimulation (Fig. 6a). Following the suppression of T-bet expression, the expression of Th2-associated genes was upregulated and IFN- $\gamma$ was downregulated at $48 \mathrm{~h}$ after stimulation (Fig. 6a). We further confirmed that various NAs other than non-CpG also induced the suppression of T-bet and the upregulation of GATA-3 and IL-4 expression (Supplementary Fig. 5a). We then compared the kinetics of the expression of Th1/Th2-associated genes upon stimulation with IL-4 or non-CpG. GATA-3 was quickly induced in $\mathrm{CD}^{+} \mathrm{T}$ cells by exogenous IL-4 (at $24 \mathrm{~h}$ ), followed by the induction of Th2 cytokines and the inhibition of T-bet and IFN- $\gamma$ expression. By contrast, in $\mathrm{CD}_{4}{ }^{+} \mathrm{T}$ cells stimulated with non-CpG, GATA-3 expression was induced after inhibition of T-bet expression, suggesting that Th2-associated genes are indirectly induced by non-CpG-mediated costimulation. In addition to these kinetic differences, inhibition of T-bet expression by non-CpG was more rapid and robust than by exogenous IL-4 (Fig. 6b). T-bet inhibits Th2 differentiation by directly inhibiting the expression of Th2 cytokines and sequestering GATA-3 from the promoters of Th2 cytokines $^{33}$. Using ChIP analysis, we showed that the binding of GATA-3 to the IL-4 and IL-13 promoters was enhanced in $\mathrm{CD}^{+} \mathrm{T}$ cells by stimulation with non-CpG (Fig. 6c). Therefore, it is likely that NA-mediated costimulation induces Th2 differentiation primarily by inhibiting T-bet expression.

To test this hypothesis further, we compared Th2 differentiation by the blockade of IFN- $\gamma$ signalling and non-CpG stimulation, because the expression of T-bet is controlled by IFN- $\gamma$ signalling ${ }^{34}$. We found that the T-bet expression in CD4 ${ }^{+}$ $\mathrm{T}$ cells cultured in the presence of anti-IFN- $\gamma$ was much lower than those stimulated with non-CpG at early time point $(48 \mathrm{~h})$ after TCR stimulation (Fig. 6d). However, the expression of GATA-3 and Th2 cytokines in T cells cultured with anti-IFN- $\gamma$ was much lower than those stimulated with non-CpG (Fig. 6d), suggesting that inhibition of T-bet expression by non-CpG is not sufficient, though partially contributes, for the upregulation of Th2-associated gene expression. Additional signal(s) are required for the induction of Th2-associated genes by non-CpG-mediated costimulation. Consistently, the Th2 polarization by the presence of anti-IFN- $\gamma$ was weaker than by non-CpG later (day 6) after TCR priming (Fig. 6e). Thus, non-CpG-mediated costimulation simultaneously inhibits $\mathrm{T}$-bet expression and enhances the expression of Th2-associated genes.

As T-bet controls Th1 development by directly activating IFN- $\gamma$ (ref. 34), it is likely that NA-mediated costimulation would inhibit Th1 development under Th1-polarizing conditions. As expected, non-CpG inhibits Th1 development and 
a

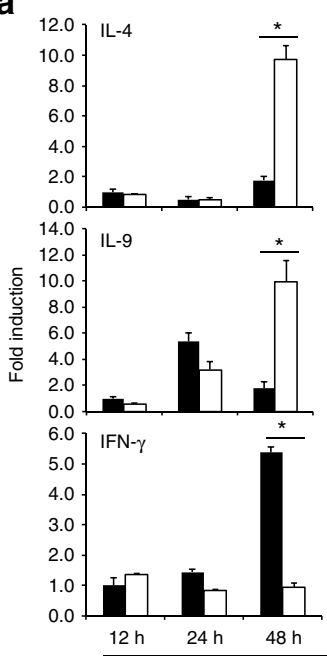

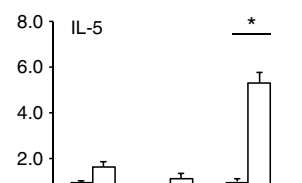
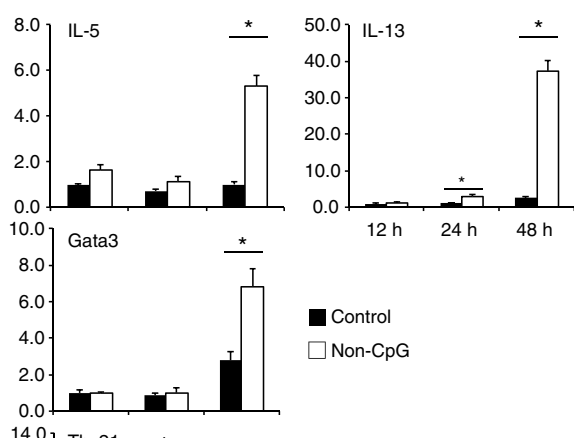

- Control

$\square$ Non-CpG

14.0 Tbx21

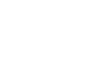

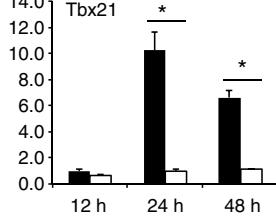

c

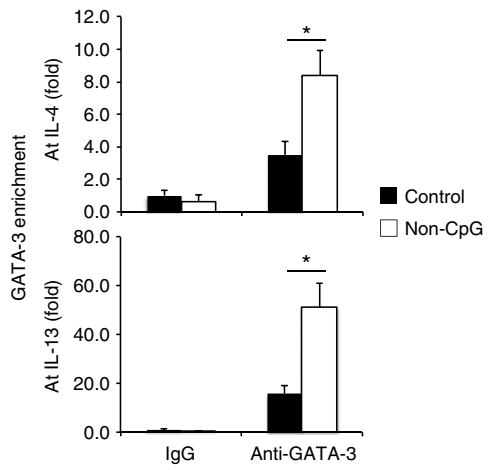

b

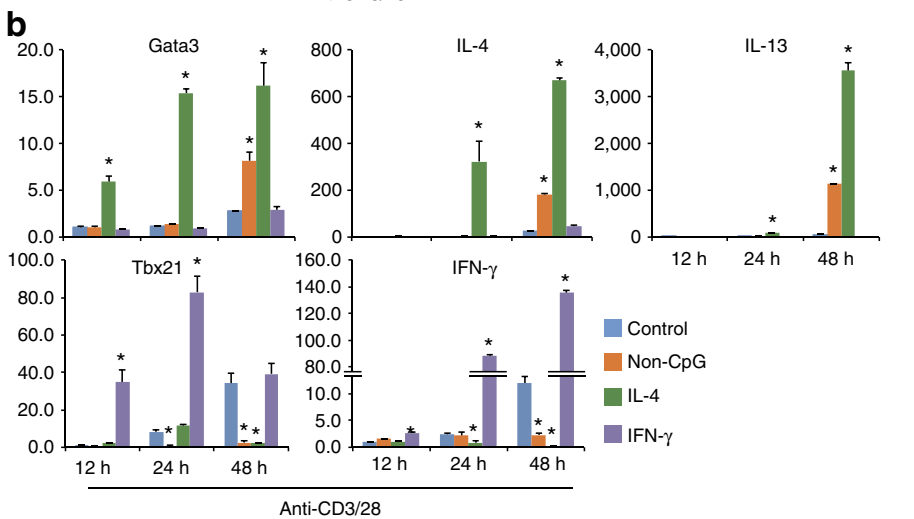

\section{d}

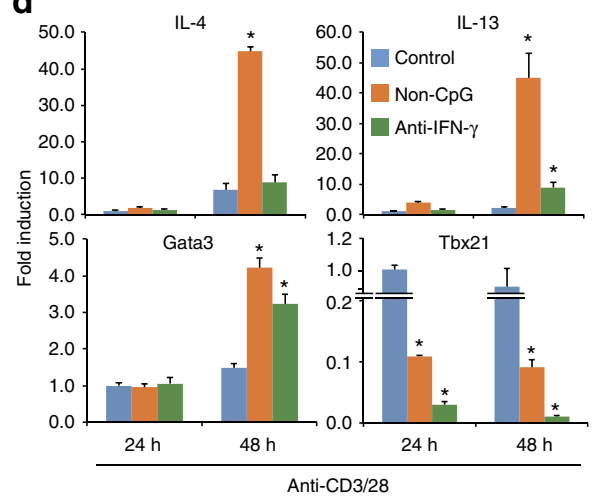

e
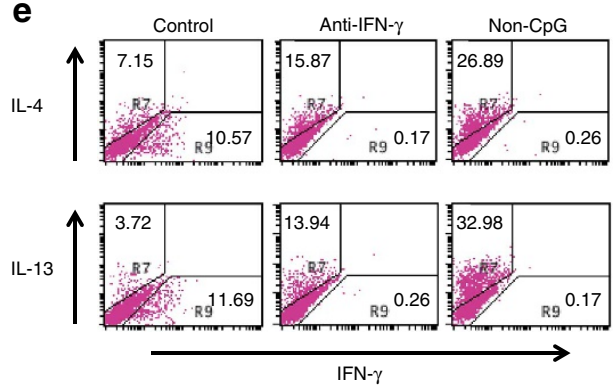

$\mathbf{f}_{12}$

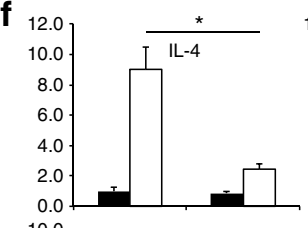

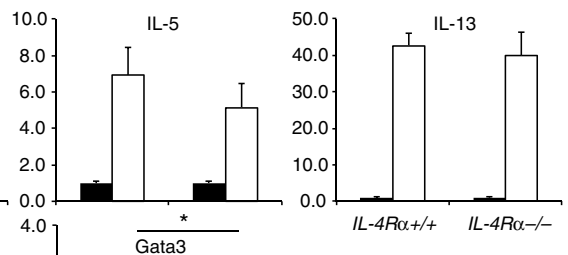

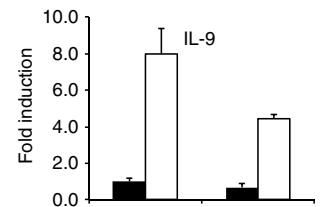

Gata3

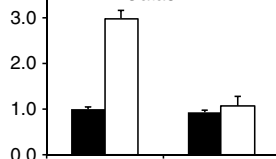

Control

$\square$ Non-CpG

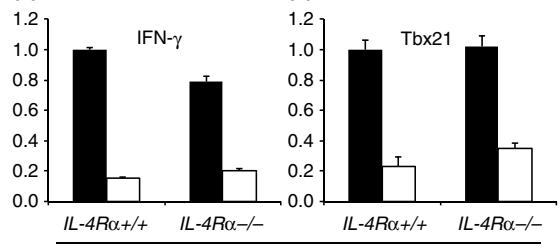

Anti-CD3/28 (48 h)

Figure 6 | Molecular mechanism of NA-mediated Th2 differentiation. (a) Naive CD4 ${ }^{+}{ }^{T}$ cells were stimulated with anti-CD3€ plus anti-CD28 with or without non-CpG for the indicated periods and mRNA expression was analysed by real-time PCR. ${ }^{*} P<0.01$, Student's $t$-test (compared with anti-CD3/CD28 alone). (b) Naive CD4 ${ }^{+}$T cells were stimulated by NAs or IL-4 and analysed similarly in (a). ${ }^{\star} P<0.01$, Student's $t$-test (compared with anti-CD3/CD28 alone). (c) Naive CD4 ${ }^{+}$T cells were stimulated with anti-CD3€ plus anti-CD28 together with non-CpG for $48 \mathrm{~h}$, and ChIP analyses were performed by immunoprecipitation with control Ab (lgG) or anti-GATA-3. Quantitative PCR analysis of the GATA3 binding at the IL-4 and IL-13 gene promoters. The results were normalized to those of a standardized aliquot of input chromatin. ${ }^{\star} P<0.05$, Student's $t$-test (compared with anti-CD3/CD28 alone). (d,e) Naive CD4 ${ }^{+}$T cells were stimulated in the presence of non-CpG or anti-IFN- $\gamma$ Ab and mRNA expression (d) and intracellular cytokine expression (day 6) (e) were analysed. ${ }^{*} P<0.01$, Student's $t$-test (compared with anti-CD3/CD28 alone).

(f) Naive $\mathrm{CD}^{+}{ }^{+}$T cells from WT and IL-4R $\alpha^{-/}$mice were stimulated and analysed similarly in $(\mathbf{a}) .{ }^{\star} P<0.01$, Student's $t$-test (compared with anti-CD3/CD28 plus non-CpG in WT cells). Error bars indicate s.d. Data are representative of at least two independent experiments. 
IFN- $\gamma$ production even under Th1 differentiation condition (Supplementary Fig. 5b).

We then analysed whether IL-4 signalling is required for the early expression of Th2-associated genes after NA-mediated costimulation. Interestingly, the augmentation of the expression of IL-4 and GATA-3 by non-CpG was severely impaired in IL-4R $\alpha$-deficient CD4 ${ }^{+}$T cells, whereas the upregulation of IL-5, IL-9 and IL-13 expression and downregulation of IFN- $\gamma$ and T-bet expression was largely unaffected (Fig. 6f). Similar results were obtained in the Stat ${ }^{-1-} \mathrm{CD} 4^{+} \mathrm{T}$ cells (Supplementary Fig. 5c). These data suggest that non-CpG-mediated T-cell costimulation directly induced the expression of IL-5, IL-9 and IL-13, partly through the inhibition of T-bet expression, whereas IL-4 and GATA-3 expression was induced by IL-4-STAT6 signalling. Naive $\mathrm{CD}^{+}{ }^{+} \mathrm{T}$ cells are capable of producing IL-4 upon primary TCR stimulation in the absence of exogenous IL-4, and the early IL-4 is rapidly consumed by the CD4 ${ }^{+}$ $\mathrm{T}$ cells themselves ${ }^{35}$. Therefore, NA-mediated IL-4 production may require early IL-4 autocrine signalling to induce the autoactivation of GATA-3 expression. Notably, however, expression of all Th2-associated genes in IL-4R $\alpha$-deficient $\mathrm{CD}^{+} \mathrm{T}$ cells disappeared by day 6 after priming with NAs. Although non-CpG stimulation induces the enrichment of GATA-3 on the IL-13 promoter (Fig. 6c), it seems that early induction of IL-13 expression by non-CpG (at $48 \mathrm{~h}$ ) is independent of GATA-3 because IL-13 expression was enhanced by non-CpG in IL- $4 \mathrm{R} \alpha$-deficient $\mathrm{CD} 4{ }^{+} \mathrm{T}$ cells despite the lack of GATA-3 upregulation by non-CpG (Fig. 6f). However, it seems that the enrichment of GATA-3 on the IL-13 promoter is critical for IL-13 expression later (in day 6) after TCR priming for its maintenance because the induction of IL-13 and GATA-3 expression by non-CpG was diminished later after TCR priming (in day 6) in IL-4R $\alpha$-deficient $\mathrm{CD} 4^{+} \mathrm{T}$ cells (Fig. $5 \mathrm{~b}, \mathrm{c}$ ). These data indicate that autocrine IL- 4 is required for the induction of IL-4 and GATA-3 upon non-CpG stimulation, which then acts to amplify and stabilize the expression of Th2-associated genes.

\section{Discussion}

The present study shows that NAs directly stimulate $\mathrm{CD} 4^{+}$ $\mathrm{T}$ cells through a NA sensor different from those of the innate system, subsequently leading to Th2 cell differentiation. In addition to TLRs, a growing number of NA sensors have been identified in the innate immune system, including RLRs, IPS-1-dependent sensors, MyD88/TRIF-dependent sensors, ASCdependent inflammasomes and STING-dependent sensors ${ }^{36}$. However, we found that recognition of NAs by $T$ cells is independent of all of these known sensors including HMGBs, although we cannot conclusively exclude the possibility that some known NA sensors work redundantly.

We found that a higher-order structure of the NAs is required for their incorporation by T cells. Since T cells can incorporate genomic self-DNA only when it is complexed with antimicrobial peptides or core histones, $\mathrm{T}$ cells may respond to NAs from dying cells at the site of inflammation and infection, where antimicrobial peptides and/or histones are released. We also demonstrated that specific recognition and uptake of RNA induces costimulatory responses. A recent study demonstrated that cells infected with several viruses including vaccinia virus contained higher-order structured RNA that stimulated MDA5 (ref. 37), indicating the possibility that $\mathrm{T}$ cells may recognize such viral RNA and are activated. Recent studies demonstrated that, upon infection with nonpermissive HIV, cytoplasmic DNA derived from incomplete reverse transcripts caused $\mathrm{CD}^{+} \mathrm{T}$ cell death through recognition of the cytosolic DNA by a sensor IFI16 followed by activation of the ASC-caspase-1 pathway ${ }^{38,39}$. It was also reported that transfected DNA was colocalized with IFI16 in activated T cells ${ }^{40}$. However, our result that DNA-mediated costimulation was normally induced in ASC-deficient $\mathrm{CD}^{+} \mathrm{T}$ cells suggests that IFI16 is not involved in the DNA-mediated costimulation in T cells. While cytosolic DNA stimulates IFI16 upon HIV infection or DNA transfection, incorporated DNAs are accumulated in endosome/lysosome and induce T-cell costimulation in our experiments without activation of IFI16 probably due to the failure to deliver DNA to the cytosol.

Furthermore, a recent study showed that immunization with RLR ligands or infection with viruses, which mainly activate RLRs, results in enhanced Th2 responses and much weaker Th1 responses $^{31}$, supporting our observation that NAs directly stimulate the induction of Th2 responses. It is known that TLR stimulation of innate immune cells promotes Th1 and Th17 responses by inducing the Th1-polarizing cytokine IL-12 and the Th17-polarizing cytokines IL-6/IL-23/IL-1 (refs 1,41). Recent studies, including ours, show that TLR2 ligands directly activate Th1 but not Th2 cells ${ }^{7}$ and promote Th17 differentiation ${ }^{8}$. In contrast to TLR, the activation of RLRs suppresses Th1 and Th17 differentiation through the inhibition of IL-12 and IL-23 production, resulting in the enhanced differentiation of Th2 cells $^{31}$. Our results demonstrate that direct stimulation of $\mathrm{T}$ cells by NAs strongly inhibit the initial expression of T-bet, which allows the initial production of IL-4 and Th2 cytokines to induce Th2 differentiation. Thus, similar to Th1 and Th17 responses induced by TLRs in innate cells and T cells, it is likely that activation of RLRs in innate cells and the NA sensor in T cells by NAs cooperatively induces Th2 differentiation.

The initial origin of IL-4 to trigger Th2 differentiation has been extensively analysed but remains unclear. It has been reported that basophils serve as Th2 cell-promoting APCs by producing IL-4 and/or thymic stromal lymphopoietin ${ }^{14}$. However, their role as APCs remains controversial ${ }^{42}$. It has been suggested that naive $\mathrm{T}$ cells are a possible source of IL-4 (refs 43,44 ), which modestly induces Th2 differentiation when IFN- $\gamma$ and IL-12 are neutralized $^{35}$. Although it has been proposed that Th2 differentiation may occur as a default pathway, $\mathrm{CD} 4{ }^{+} \mathrm{T}$ cells in IL-12 p40-deficient mice fail to differentiate into Th2 cells in response to intracellular pathogens ${ }^{45}$. Thus, the simple blocking of Th1-inducing stimuli such as IL-12 or IFN- $\gamma$ is not sufficient to induce Th2 differentiation, suggesting the existence of additional Th2-inducing factors. However, such factors for the initial triggering of IL-4 production from naive $\mathrm{CD} 44^{+} \mathrm{T}$ cells under physiological conditions have not been identified. We here provide strong evidence that the initial IL-4 production derived from naive $\mathrm{CD} 4^{+} \mathrm{T}$ cells upon recognition of NAs in the absence of any exogenous cytokines or neutralizing antibodies instructs naive $\mathrm{CD} 4^{+} \mathrm{T}$ cells to differentiate into Th2 cells.

A recent report demonstrated that aluminium hydroxide adjuvant (alum) causes cell death and release of host DNA at sites of immunization, which mediates the adjuvant effect for Th2-biased adaptive responses ${ }^{30}$. As the mechanism to induce Th2 responses, it has been reported that uric acid released in the peritoneal cavity after injection of alum may have a role in promoting Th2 cell responses independently of the ASC inflammasome or TLR signalling ${ }^{46}$. As uric acid crystals released at the sites of immunization/inflammation induce extracellular DNA traps formation by neutrophil, eosinophil and basophil ${ }^{47}$, uric acid-induced extracellular DNA traps may directly stimulate $\mathrm{T}$ cells to induce $\mathrm{Th} 2$ response. A recent study reported that defects in clearance of apoptotic airway epithelial cells upon environmental allergen encounter lead to augmented Th2 cytokine production and airway hyper-responsiveness ${ }^{48}$, indicating that in vivo Th2 responses are closely related to host 
cell death accompanied by host DNA release. In line with the hypothesis, we found that self-DNA from dead cells induces Th2 differentiation.

Collectively, we have identified NAs as a direct Th2-inducing factor, which induces initial production of IL- 4 by naive $\mathrm{CD} 4^{+}$ $\mathrm{T}$ cells, which in turn induces Th2 differentiation. Although we have not identified the NAs sensor in T cells yet, our results provide the possibility that NAs may be critical targets for the development of improved vaccine adjuvants and the overall design of therapeutics to control allergic diseases.

\section{Methods}

Mice. C57BL/6 mice were purchased from Clea Japan, Inc. The mice deficient in MyD88, TRIF-deficient, IPS-1 ZBP1 and STAT6 were provided by Dr Akira S. $\mathrm{Asc}^{-1}$ mice were provided by Dr Taniguchi S and Dr Noda T. Sting ${ }^{-1-}$ and $I_{44 a^{-/-}}$mice were provided by Dr Barber GN and Dr Brombacher F, respectively. Mice aged 8-16 weeks were used. All mice were maintained under specific pathogen-free conditions and all experiments were conducted under protocols approved by RIKEN Yokohama Institute.

ELISA and cell growth analysis. Cell culture supernatants were analysed by ELISA for the production of IL-2 (BD biosciences), IL-4 (BD biosciences) and IFN- $\gamma$ (BD biosciences). Cell growth was assessed by the MTS assay-based Cell Counting Kit-8 (DOJINDO).

Helper T-cell differentiation. $\mathrm{CD} 4^{+} / \mathrm{CD} 25^{-} / \mathrm{CD} 62 \mathrm{~L}^{+} / \mathrm{NK} 1.1^{-}$(naive) $\mathrm{T}$ cells were isolated from spleens using a FACS-Aria cell sorter. For Th0 cells, cells were stimulated with plate-bound anti-CD3 (2C11, $10 \mu \mathrm{g} \mathrm{ml}^{-1}$ ) and anti-CD28 (PV-1, $10 \mu \mathrm{g} \mathrm{ml}^{-1}$ ) Abs in the presence of the indicated ligands. For Th1 cells, cells were cultured in the presence of IL-12 $\left(10 \mathrm{ng} \mathrm{ml}^{-1}\right)$ and anti-IL-4 Abs $\left(10 \mu \mathrm{g} \mathrm{ml}^{-1}\right)$. For Th2 cells, cells were similarly cultured in the presence of IL- $4\left(10 \mathrm{ng} \mathrm{ml}^{-1}\right)$.

Real-time quantitative PCR. After removal of genomic DNA by treatment with DNase (Wako Nippon Gene), randomly primed cDNA strands were generated with reverse transcriptase II (Invitrogen). RNA expression was quantified by real-time PCR with the following gene-specific primers and values were normalized to the expression of Rps18 mRNA (Supplementary Table 2).

Reagents and Abs. The TLR2 ligands N-palmitoyl-S-(2,3-bis(palmitoyloxy)$(2 R S)$-propyl)-Cys-Ser-Lys 4 (Pam3) and macrophage-activating lipopeptide 2 (MALP-2) were purchased from EMC Microcollections. Poly(I:C), a TLR3 ligand, and LPS (Escherichia. coli O111:B4), a TLR4 ligand, were obtained from GE Healthcare Biosciences and Sigma, respectively. Flagellin, a TLR5 ligand, and loxoribine, a TLR7 ligand, were obtained from InvivoGen. Oligo DNAs including TLR9 ligands were purchased from Hokkaido System Science. Poly(A), poly(U), poly $(\mathrm{C})$, poly $(\mathrm{G})$, poly $(\mathrm{I})$, poly $(\mathrm{A}: \mathrm{U})$ and $\operatorname{poly}(\mathrm{C}: \mathrm{G})$ were purchased from Sigma. Calf thymus DNA and E. coli DNA was from Sigma and Invivogen, respectively. LL37 was from AnaSpec. Histone H1, H2A, H2B, H3 and H4 were from New England BioLabs. LL37 or histones were first premixed with genomic DNA (peptide:DNA mass ratio of 2:1). After 30-min incubation at room temperature, the mix was added to the T-cell cultures (final concentration was $10 \mu \mathrm{g} \mathrm{ml}^{-1}$ of DNA).

Abs specific for anti-IL-4 PE (11B11, 1:25 dilution) and anti-IFN- $\gamma$ FITC (XMG1.2, 1:25 dilution) were obtained from BD Biosciences; anti-IL-5 PE (TRFK5, 1:25 dilution), anti-IL-10 PE (1:25 dilution) and anti-IL-13 PE (eBio13A, 1:25 dilution) from eBioscience; anti-IL-9 PE (RM9A4, 1:25 dilution) from BioLegend. ChIP analysis used mAb to GATA-3 (HG3-31AC; Santa Cruz, 1:125 dilution).

Intracellular cytokine staining analysis. $\mathrm{CD} 4{ }^{+} \mathrm{T}$ cells were restimulated with immobilized anti-CD3 and anti-CD28 for $6 \mathrm{~h}$ in the presence of $2 \mu \mathrm{M}$ monensin (Sigma, St Louis, MO). Cells were fixed with $4 \%$ paraformaldehyde and permeabilized with $0.5 \%$ Triton X-100. After blocking with 3\% BSA-PBS, cells were stained with antibodies to each cytokine. Flow cytometric analysis was performed on a FACSCalibur and data were analysed with BD CellQuest.

Cellular uptake of NA. To analyse the uptake of NA, $2 \times 10^{5}$ cells were preincubated at $37^{\circ} \mathrm{C}$ for $10 \mathrm{~min}$ in medium. Cells were incubated with fluorescencelabelled NA at $37^{\circ} \mathrm{C}$ for $90 \mathrm{~min}$. Then cells were washed once in HANKS $/ 0.1 \%$ BSA followed by an acidic wash with $100 \mathrm{mM}$ acetic acid, $150 \mathrm{mM} \mathrm{NaCl}$ (pH2.7) for $1 \mathrm{~min}$ to remove unbound and cell surface-bound NA. Subsequently, cells were washed two times in HANKS/0.1\% BSA, and were analysed by flow cytometry using the FACSCalibur.

Reporter cells. The 2B4-NFAT-GFP cells have been described ${ }^{49}$ and the $2 \mathrm{~B} 4-\mathrm{NF}-$ $\mathrm{kB}-\mathrm{GFP}$ cells were established by transfection of NF- $\mathrm{kB}-\mathrm{GFP}$ into $2 \mathrm{~B} 4$ hybridoma cells. These cells were cultured in RPMI 1640 medium supplemented with $10 \%$ (vol/vol) FCS and $\beta$-mercaptoethanol.

Chromatin immunoprecipitation assay. Cells were fixed for $10 \mathrm{~min}$ at $4{ }^{\circ} \mathrm{C}$ with $10 \%$ formaldehyde. After incubation, glycine was added to a final concentration of $0.125 \mathrm{M}$ to quench the formaldehyde. Cells were pelleted, washed three times with ice-cold PBS and then lysed. The lysates were sonicated to reduce DNA length to between 200 and 300 base pairs. The chromatin was pre-cleared with protein G agarose beads for $6 \mathrm{~h}$ and then incubated with $4 \mu \mathrm{g}$ of anti-GATA-3 (HG3-31) agarose conjugate antibody (Santa Cruz, sc-268 AC) or control IgG overnight. The precipitates were washed and eluted in $120 \mu \mathrm{l}$ of $\mathrm{NaHCO}_{3}$ buffer with $1 \%$ SDS. The samples were treated with RNase and Proteinase $\mathrm{K}$ and then de-crosslinked at $65^{\circ} \mathrm{C}$ overnight. Precipitated DNA was further purified with Qiaquick PCR purification kit (Qiagen) and was analysed by quantitative PCR (Supplementary Table 2$)^{50}$

Confocal microscopic imaging. Cells were settled on glass-bottom, 35-mm tissue culture dishes (MATSUNAMI GLASS). Confocal microscopy analyses were performed with a Leica TCS SP5 confocal microscope with an oil immersion objective (HCX PL APO × 63/1.40-0.60 NA, Leica). Dual-color images were acquired using a sequential acquisition mode to avoid cross-excitation. To visualize the Histonecalf thymus DNA complex in $\mathrm{CD} 4^{+} \mathrm{T}$ cells, calf thymus DNA (Sigma) was labelled with Cy5 using the Label IT Nucleic Acid Labelling Reagents (Mirus) and histone H3 was labelled with DyLight 488 using the antibody labelling kit (Pierce), according to the standard protocol provided by the respective manufacturers.

RNA interference. Double-stranded oligonucleotides corresponding to the target sequences were cloned into the pSuper.Retro RNAi plasmid (OligoengineInc.). The siRNA targeting sequences which function for commonly all three murine HMGB1/2/3 are $5^{\prime}$-GAGAAGTATGAGAAGGATATT- ${ }^{\prime}$ and $5^{\prime}$-AAGTATGAGA AGGATATTGCT- $3^{\prime}$

Statistics. Statistical significance was determined by a two-tailed unpaired Student's $t$-test. $P<0.05$ was considered statistically significant.

\section{References}

1. Takeda, K., Kaisho, T. \& Akira, S. Toll-like receptors. Annu. Rev. Immunol. 21 335-376 (2003).

2. Medzhitov, R. Toll-like receptors and innate immunity.. Nat. Rev. Immunol. 1, 135-145 (2001).

3. Komai-Koma, M., Jones, L., Ogg, G. S., Xu, D. \& Liew, F. Y. TLR2 is expressed on activated T cells as a costimulatory receptor. Proc. Natl Acad. Sci. USA 101, 3029-3034 (2004).

4. Cottalorda, A. et al. TLR2 engagement on CD8 T cells lowers the threshold for optimal antigen-induced T cell activation. Eur. J. Immunol. 36, 1684-1693 (2006).

5. Sutmuller, R. P. et al. Toll-like receptor 2 controls expansion and function of regulatory T cells. J. Clin. Invest. 116, 485-494 (2006).

6. Liu, H., Komai-Koma, M., Xu, D. \& Liew, F. Y. Toll-like receptor 2 signaling modulates the functions of CD $4+\mathrm{CD} 25+$ regulatory T cells. Proc. Natl Acad. Sci. U. S. A. 103, 7048-7053 (2006).

7. Imanishi, T. et al. Cutting edge: TLR2 directly triggers Th1 effector functions. J. Immunol. 178, 6715-6719 (2007).

8. Reynolds, J. M. et al. Toll-like receptor 2 signaling in CD4(+) T lymphocytes promotes $\mathrm{T}$ helper 17 responses and regulates the pathogenesis of autoimmune disease. Immunity 32, 692-702 (2010).

9. Caron, G. et al. Direct stimulation of human T cells via TLR5 and TLR7/8: flagellin and R-848 up-regulate proliferation and IFN-gamma production by memory CD4 + T cells. J. Immunol. 175, 1551-1557 (2005).

10. Gelman, A. E. et al. The adaptor molecule MyD88 activates PI-3 kinase signaling in $\mathrm{CD} 4+\mathrm{T}$ cells and enables $\mathrm{CpG}$ oligodeoxynucleotide-mediated costimulation. Immunity 25, 783-793 (2006).

11. Gelman, A. E., Zhang, J., Choi, Y. \& Turka, L. A. Toll-like receptor ligands directly promote activated CD4 + T cell survival. J. Immunol. 172, 6065-6073 (2004).

12. Peng, G. et al. Toll-like receptor 8-mediated reversal of $\mathrm{CD} 4+$ regulatory T cell function. Science 309, 1380-1384 (2005).

13. Zhu, J., Yamane, H. \& Paul, W. E. Differentiation of effector CD4 T cell populations $\left({ }^{*}\right)$. Annu. Rev. Immunol. 28, 445-489 (2010).

14. Paul, W. E. \& Zhu, J. How are $\mathrm{T}(\mathrm{H}) 2$-type immune responses initiated and amplified? Nat. Rev. Immunol. 10, 225-235 (2010).

15. Kerkmann, M. et al. Spontaneous formation of nucleic acid-based nanoparticles is responsible for high interferon-alpha induction by $\mathrm{CpG}-\mathrm{A}$ in plasmacytoid dendritic cells. J. Biol. Chem. 280, 8086-8093 (2005).

16. Bishop, J. S. et al. Intramolecular G-quartet motifs confer nuclease resistance to a potent anti-HIV oligonucleotide. J. Biol. Chem. 271, 5698-5703 (1996). 
17. Dalpke, A. H., Zimmermann, S., Albrecht, I. \& Heeg, K. Phosphodiester CpG oligonucleotides as adjuvants: polyguanosine runs enhance cellular uptake and improve immunostimulative activity of phosphodiester CpG oligonucleotides in vitro and in vivo. Immunology 106, 102-112 (2002).

18. Arnott, S., Chandrasekaran, R. \& Marttila, C. M. Structures for polyinosinic acid and polyguanylic acid. Biochem. J. 141, 537-543 (1974).

19. Lande, R. et al. Plasmacytoid dendritic cells sense self-DNA coupled with antimicrobial peptide. Nature 449, 564-569 (2007).

20. Mantovani, A., Cassatella, M. A., Costantini, C. \& Jaillon, S. Neutrophils in the activation and regulation of innate and adaptive immunity. Nat. Rev. Immunol. 11, 519-531 (2011).

21. Takaoka, A. et al. DAI (DLM-1/ZBP1) is a cytosolic DNA sensor and an activator of innate immune response. Nature 448, 501-505 (2007).

22. Hornung, V. et al. AIM2 recognizes cytosolic dsDNA and forms a caspase-1activating inflammasome with ASC. Nature 458, 514-518 (2009).

23. Ishikawa, H. \& Barber, G. N. STING is an endoplasmic reticulum adaptor that facilitates innate immune signalling. Nature 455, 674-678 (2008).

24. Ishikawa, H., Ma, Z. \& Barber, G. N. STING regulates intracellular DNA-mediated, type I interferon-dependent innate immunity. Nature 461, 788-792 (2009).

25. Ishii, K. J. et al. A Toll-like receptor-independent antiviral response induced by double-stranded B-form DNA. Nat. Immunol. 7, 40-48 (2006).

26. Kato, H. et al. Length-dependent recognition of double-stranded ribonucleic acids by retinoic acid-inducible gene-I and melanoma differentiation-associated gene 5. J. Exp. Med. 205, 1601-1610 (2008).

27. Kawai, T. et al. IPS-1, an adaptor triggering RIG-I- and Mda5-mediated type I interferon induction. Nat. Immunol. 6, 981-988 (2005).

28. Yanai, H. et al. HMGB proteins function as universal sentinels for nucleic-acidmediated innate immune responses. Nature 462, 99-103 (2009).

29. Smith-Garvin, J. E., Koretzky, G. A. \& Jordan, M. S. T cell activation. Annu. Rev. Immunol. 27, 591-619 (2009).

30. Marichal, T. et al. DNA released from dying host cells mediates aluminum adjuvant activity. Nat. Med. 17, 996-1002 (2011).

31. Negishi, H. et al. Cross-interference of RLR and TLR signaling pathways modulates antibacterial T cell responses. Nat. Immunol. 13, 659-666 (2012).

32. Zheng, W. \& Flavell, R. A. The transcription factor GATA-3 is necessary and sufficient for Th2 cytokine gene expression in CD4 T cells. Cell 89, 587-596 (1997).

33. Hwang, E. S., Szabo, S. J., Schwartzberg, P. L. \& Glimcher, L. H. T helper cell fate specified by kinase-mediated interaction of T-bet with GATA-3. Science 307, 430-433 (2005).

34. Afkarian, M. et al. T-bet is a STAT1-induced regulator of IL-12R expression in naive CD4 + T cells. Nat. Immunol. 3, 549-557 (2002).

35. Noben-Trauth, N., Hu-Li, J. \& Paul, W. E. Conventional, naive CD4 + T cells provide an initial source of IL-4 during Th2 differentiation. J. Immunol. 165, 3620-3625 (2000).

36. Desmet, C. J. \& Ishii, K. J. Nucleic acid sensing at the interface between innate and adaptive immunity in vaccination. Nat. Rev. Immunol. 12, 479-491 (2012).

37. Pichlmair, A. et al. Activation of MDA5 requires higher-order RNA structures generated during virus infection. J. Virol. 83, 10761-10769 (2009).

38. Monroe, K. M. et al. IFI16 DNA sensor is required for death of lymphoid CD4 T cells abortively infected with HIV. Science 343, 428-432 (2014).

39. Doitsh, G. et al. Cell death by pyroptosis drives CD4 T-cell depletion in HIV-1 infection. Nature 505, 509-514 (2014).

40. Berg, R. K. et al. T cells detect intracellular DNA but fail to induce Type I IFN responses: implications for restriction of HIV replication. PloS One 9, e84513 (2014).
41. Goriely, S., Neurath, M. F. \& Goldman, M. How microorganisms tip the balance between interleukin-12 family members. Nat. Rev. Immunol. 8, 81-86 (2008).

42. Kim, S. et al. Cutting edge: basophils are transiently recruited into the draining lymph nodes during helminth infection via IL-3, but infection-induced Th2 immunity can develop without basophil lymph node recruitment or IL-3. J. Immunol. 184, 1143-1147 (2010).

43. Yagi, R. et al. The IL-4 production capability of different strains of naive $\mathrm{CD} 4(+) \mathrm{T}$ cells controls the direction of the $\mathrm{T}(\mathrm{h})$ cell response. Int. Immunol. 14, 1-11 (2002).

44. Liu, Z. et al. IL-2 and autocrine IL-4 drive the in vivo development of antigenspecific Th2 T cells elicited by nematode parasites. J. Immunol. 174, 2242-2249 (2005).

45. Jankovic, D. et al. In the absence of IL-12, CD4 $(+)$ T cell responses to intracellular pathogens fail to default to a Th2 pattern and are host protective in an IL-10(-/-) setting. Immunity 16, 429-439 (2002).

46. Kool, M. et al. An unexpected role for uric acid as an inducer of T helper 2 cell immunity to inhaled antigens and inflammatory mediator of allergic asthma. Immunity 34, 527-540 (2011).

47. Schorn, C. et al. Monosodium urate crystals induce extracellular DNA traps in neutrophils, eosinophils, and basophils but not in mononuclear cells. Front. Immunol. 3, 277 (2012).

48. Juncadella, I. J. et al. Apoptotic cell clearance by bronchial epithelial cells critically influences airway inflammation. Nature 493, 547-551 (2013).

49. Ohtsuka, M. et al. NFAM1, an immunoreceptor tyrosine-based activation motif-bearing molecule that regulates B cell development and signaling. Proc. Natl Acad. Sci. USA 101, 8126-8131 (2004).

50. Tanaka, S. et al. The enhancer HS2 critically regulates GATA-3-mediated Il4 transcription in $\mathrm{T}(\mathrm{H}) 2$ cells. Nat. Immunol. 12, 77-85 (2011).

\section{Acknowledgements}

We thank S. Yamasaki, T. Yokosuka, S. Tsukumo, A. Takeuchi, R. Onishi, H. Ike, Y. Motomura and M. Kubo for discussions and experimental help, M. Sakuma, M. Unno and A. Fujii for technical support, and H. Yamaguchi, M. Yoshioka and S. Kato for secretarial assistance. This work was supported by a Grant-in-Aid for Scientific Research from the Ministry of Education, Culture, Sports, Science and Technology of Japan (JSPS KAKENHI Grant numbers 24790489 for T.I. and 24229004 for T.S.).

\section{Authors contributions}

T.I. designed and performed the experiments and wrote the paper; C.I., M.E.S.G.B., Y.K A.H-T. and H.H performed the experiments; T.K., O.T., K.J.I., S.T., T.N., F.B., G.N.B. and S.A. provided knockout mice; T.S. designed the experiments and wrote the paper.

\section{Additional information}

Supplementary Information accompanies this paper at http://www.nature.com/ naturecommunications

Competing financial interests: The authors declare no competing financial interests.

Reprints and permission information is available online at http://npg.nature.com/ reprintsandpermissions/

How to cite this article: Imanishi, T. et al. Nucleic acid sensing by T cells initiates Th2 cell differentiation. Nat. Commun. 5:3566 doi: 10.1038/ncomms4566 (2014). 